PROCEEDINGS OF THE

AMERICAN MATHEMATICAL SOCIETY

Volume 137, Number 4, April 2009, Pages 1223-1233

S 0002-9939(08)09653-6

Article electronically published on October 16, 2008

\title{
PRODUCING SET-THEORETIC COMPLETE INTERSECTION MONOMIAL CURVES IN $\mathbb{P}^{n}$
}

\author{
MESUT ŞAHIN \\ (Communicated by Ted Chinburg)
}

\begin{abstract}
In this paper we describe an algorithm for producing infinitely many examples of set-theoretic complete intersection monomial curves in $\mathbb{P}^{n+1}$, starting with a single set-theoretic complete intersection monomial curve in $\mathbb{P}^{n}$. Moreover we investigate the numerical criteria to decide when these monomial curves can or cannot be obtained via semigroup gluing.
\end{abstract}

\section{INTRODUCTION}

It is well known that a variety in an $n$-space can be written as the intersection of $n$ hypersurfaces set theoretically; see [5. It is then natural to ask whether this number is minimal. A curve in $n$-space which is the intersection of $n-1$ hypersurfaces is called a set-theoretic complete intersection, s.t.c.i. for short. If moreover its defining ideal is generated by $n-1$ polynomials, then it is called an ideal theoretic complete intersection, abbreviated i.t.c.i. Determining set-theoretic or ideal-theoretic complete intersection curves is a classical and long-standing problem in algebraic geometry. An associated problem is to give explicitly the equations of the hypersurfaces involved. When the characteristic of the field $K$ is positive, it is known that all monomial curves are s.t.c.i.'s in $\mathbb{P}^{n}$; see 8]. However the question is still open in the characteristic zero case despite the tremendous progress in this direction; see for example [6, 7, 16] and the references there for some recent activity.

The purpose of the present paper is to describe a method to produce infinitely many s.t.c.i. monomial curves starting from one single s.t.c.i. monomial curve; see section 4. Our approach has the side novelty of describing explicitly the equations of hypersurfaces on which these new monomial curves lie as an s.t.c.i. On the other hand, semigroup gluing being one of the most popular techniques of recent research, we develop numerical criteria to determine when these new curves can or cannot be obtained via gluing; see section 3. In the last section we discuss several consequences and variations of these results.

\section{Preliminaries}

Throughout the paper, $K$ will be assumed to be an algebraically closed field of characteristic zero. By an affine monomial curve $C\left(m_{1}, \ldots, m_{n}\right)$, for some positive

Received by the editors May 29, 2007, and, in revised form, June 1, 2007, October 11, 2007, March 4, 2008, and April 15, 2008.

2000 Mathematics Subject Classification. Primary 14M10; Secondary 14H45.

Key words and phrases. Set-theoretic complete intersections, monomial curves.

(C) 2008 American Mathematical Society Reverts to public domain 28 years from publication 
integers $m_{1}<\cdots<m_{n}$ with $\operatorname{gcd}\left(m_{1}, \ldots, m_{n}\right)=1$, we mean a curve with generic zero $\left(v^{m_{1}}, \ldots, v^{m_{n}}\right)$ in the affine $n$-space $\mathbb{A}^{n}$, over $K$. By a projective monomial curve $\bar{C}\left(m_{1}, \ldots, m_{n}\right)$ we mean a curve with generic zero

$$
\left(u^{m_{n}}, u^{m_{n}-m_{1}} v^{m_{1}}, \ldots, u^{m_{n}-m_{n-1}} v^{m_{n-1}}, v^{m_{n}}\right)
$$

in the projective $n$-space $\mathbb{P}^{n}$, over $K$. Note that $\bar{C}\left(m_{1}, \ldots, m_{n}\right)$ is the projective closure of $C\left(m_{1}, \ldots, m_{n}\right)$.

Whenever we write $\bar{C} \subset \mathbb{P}^{n}$ to simplify the notation, we always mean a monomial curve $\bar{C}\left(m_{1}, \ldots, m_{n}\right)$ for some fixed positive integers $m_{1}<\cdots<m_{n}$ with $\operatorname{gcd}\left(m_{1}, \ldots, m_{n}\right)=1$.

Let $m$ be a positive integer in the numerical semigroup generated by $m_{1}, \ldots, m_{n}$; i.e. $m=s_{1} m_{1}+\cdots+s_{n} m_{n}$, where $s_{1}, \ldots, s_{n}$ are some non-negative integers. Note that in general there is no unique choice for $s_{1}, \ldots, s_{n}$ to represent $m$ in terms of $m_{1}, \ldots, m_{n}$. We define the degree $\delta(m)$ of $m$ to be the minimum of all possible sums $s_{1}+\cdots+s_{n}$. If $\ell$ is a positive integer with $\operatorname{gcd}(\ell, m)=1$, then we say that the monomial curve $\bar{C}\left(\ell m_{1}, \ldots, \ell m_{n}, m\right)$ in $\mathbb{P}^{n+1}$ is an extension of $\bar{C}$. We similarly define $C\left(\ell m_{1}, \ldots, \ell m_{n}, m\right)$ to be an extension of $C=C\left(m_{1}, \ldots, m_{n}\right)$. We say that an extension is nice if $\delta(m)>\ell$ and bad otherwise, adopting the terminology of 1 .

When the integers $m_{1}, \ldots, m_{n}$ are fixed and understood in a discussion, we will use $\bar{C}_{\ell, m}$ to denote the extensions $\bar{C}\left(\ell m_{1}, \ldots, \ell m_{n}, m\right)$ in $\mathbb{P}^{n+1}$ and use $C_{\ell, m}$ to denote the extensions $C\left(\ell m_{1}, \ldots, \ell m_{n}, m\right)$ in $\mathbb{A}^{n+1}$.

2.1. Extensions of monomial curves in $\mathbb{A}^{n}$. Let $C=C\left(m_{1}, \ldots, m_{n}\right)$ be an s.t.c.i. monomial curve in $\mathbb{A}^{n}$. In this section, we show that all extensions of $C$, in the sense defined above, are s.t.c.i. For this we first define, for any ideal $I \subset K\left[x_{1}, \ldots, x_{n+1}\right], \Gamma_{\ell}(I)$ to be the ideal which is generated by all polynomials of the form $\Gamma_{\ell}(g)$, where $\Gamma_{\ell}\left(g\left(x_{1}, \ldots, x_{n+1}\right)\right)=g\left(x_{1}, \ldots, x_{n}, x_{n+1}^{\ell}\right)$, for all $g \in I$. We use the following trick of M. Morales:

Lemma 2.1 (9, Lemma 3.2]). Let $Y_{\ell}$ be the monomial curve $C\left(\ell m_{1}, \ldots, \ell m_{n}, m_{n+1}\right)$ in $\mathbb{A}^{n+1}$. Then $I\left(Y_{\ell}\right)=\Gamma_{\ell}\left(I\left(Y_{1}\right)\right)$.

For any extension of $C$ of the form $C_{\ell, m}$, we obviously have $I(C) \subset I\left(C_{\ell, m}\right)$ and $I\left(C_{\ell, m}\right) \cap K\left[x_{1}, \ldots, x_{n}\right]=I(C)$. The exact relation between the ideals of $C$ and $C_{\ell, m}$ are given by the following lemma.

Lemma 2.2. Let $m=s_{1} m_{1}+\cdots+s_{n} m_{n}$. For any positive integer $\ell$ with $\operatorname{gcd}(\ell, m)=1$ we have $I\left(C_{\ell, m}\right)=I(C)+(G)$, where $G=x_{1}{ }^{s_{1}} \cdots x_{n}{ }^{s_{n}}-x_{n+1}^{\ell}$.

Proof.

Case $\ell=1$ : We show that $I\left(C_{1, m}\right)=I(C)+\left(x_{1}{ }^{s_{1}} \cdots x_{n}{ }^{s_{n}}-x_{n+1}\right)$.

For any polynomial $f \in K\left[x_{1}, \ldots, x_{n+1}\right]$, there are polynomials $g \in K\left[x_{1}, \ldots, x_{n}\right]$ and $h \in K\left[x_{1}, \ldots, x_{n+1}\right]$ such that

$$
\begin{aligned}
f\left(x_{1}, \ldots, x_{n+1}\right) & =f\left(x_{1}, \ldots, x_{n}, x_{n+1}-x_{1}^{s_{1}} \cdots x_{n}^{s_{n}}+x_{1}^{s_{1}} \cdots x_{n}^{s_{n}}\right) \\
& =g\left(x_{1}, \ldots, x_{n}\right)+\left(x_{1}^{s_{1}} \cdots x_{n}^{s_{n}}-x_{n+1}\right) h\left(x_{1}, \ldots, x_{n+1}\right) .
\end{aligned}
$$

This identity implies that $f \in I\left(C_{1, m}\right)$ if and only if $g \in I(C)$.

Case $\ell>1$ : Applying Lemma 2.1 with $Y_{1}=C_{1, m}$ we have

$$
\begin{aligned}
I\left(C_{\ell, m}\right) & =\Gamma_{\ell}\left(I\left(C_{1, m}\right)\right) \text { by Lemma } 2.1 \\
& =\Gamma_{\ell}\left(I(C)+\left(x_{1}^{s_{1}} \cdots x_{n}^{s_{n}}-x_{n+1}\right)\right) \text { by the first part of this lemma } \\
& =I(C)+(G) .
\end{aligned}
$$


This lemma provides an alternate proof to the following theorem, which is a special case of [16, Theorem 2].

Theorem 2.3. If $C \subset \mathbb{A}^{n}$ is an s.t.c.i. monomial curve, then all extensions of the form $C_{\ell, m} \subset \mathbb{A}^{n+1}$ are also s.t.c.i. monomial curves.

Proof. Since $I\left(C_{\ell, m}\right)=I(C)+(G)$ by Lemma 2.2, it follows that

$$
\begin{aligned}
Z\left(I\left(C_{\ell, m}\right)\right) & =Z(I(C)+(G)), \\
C_{\ell, m} & =Z(I(C)) \cap Z(G),
\end{aligned}
$$

where $Z(\cdot)$ denotes the zero set as usual. Hence $C_{\ell, m}$ is an s.t.c.i. if $C$ is also.

\section{Extensions that CANnOt Be obtained By GLuing}

If $\bar{C}\left(m_{1}, \ldots, m_{n+1}\right)$ is a monomial curve in $\mathbb{P}^{n+1}$, then there is a corresponding semigroup $\mathbb{N} T$, where

$$
T=\left\{\left(m_{n+1}, 0\right),\left(m_{n+1}-m_{1}, m_{1}\right), \ldots,\left(m_{n+1}-m_{n}, m_{n}\right),\left(0, m_{n+1}\right)\right\} \subset \mathbb{N}^{2} .
$$

Let $T=T_{1} \sqcup T_{2}$ be a decomposition of $T$ into two disjoint proper subsets. Without loss of generality assume that the cardinality of $T_{1}$ is less than or equal to the cardinality of $T_{2}$. $\mathbb{N} T$ is called a gluing of $\mathbb{N} T_{1}$ and $\mathbb{N} T_{2}$ if there exists a non-zero $\alpha \in \mathbb{N} T_{1} \cap \mathbb{N} T_{2}$ such that $\mathbb{Z} \alpha=\mathbb{Z} T_{1} \cap \mathbb{Z} T_{2}$. Following the literature we write $I(T)$ for the ideal of the toric variety corresponding to the affine semigroup $\mathbb{N} T$. Note that if $\mathbb{N} T$ is a gluing of $\mathbb{N} T_{1}$ and $\mathbb{N} T_{2}$, then we have $I(T)=I\left(T_{1}\right)+I\left(T_{2}\right)+\left(G_{\alpha}\right)$, where $G_{\alpha}$ is the relation polynomial; see [16].

We note that the condition $\mathbb{Z} \alpha=\mathbb{Z} T_{1} \cap \mathbb{Z} T_{2}$ is not fulfilled when $T_{1}$ is not a singleton. Hence we formulate this observation to be the following:

Proposition 3.1. If $T_{1}$ is not a singleton, then $\mathbb{N} T$ is not a gluing of $\mathbb{N} T_{1}$ and $\mathbb{N} T_{2}$.

Proof. If $T_{1}$ is not a singleton, then neither is $T_{2}$ by the assumption on the cardinalities of these sets. Thus $\mathbb{Z} T_{1}$ and $\mathbb{Z} T_{2}$ are submodules of $\mathbb{Z}^{2}$ of rank two each. It is elementary to show that their intersection has rank two. For instance, let $r$ and $t$ be generators of $\mathbb{Z} T_{1}$. Then the images of $r$ and $t$ have finite order in the finite group $\mathbb{Z}^{2} / \mathbb{Z} T_{2}$, meaning that $a r$ and $b t$ are in $\mathbb{Z} T_{2}$ for some positive integers $a$ and $b$. Then the rank two $\mathbb{Z}$-module generated by $a r$ and $b t$ is contained in the intersection $\mathbb{Z} T_{1} \cap \mathbb{Z} T_{2}$, which must be of rank two itself, being a submodule of $\mathbb{Z}^{2}$.

Hence the intersection cannot be generated by a single element. Thus $\mathbb{N} T$ is not a gluing of $\mathbb{N} T_{1}$ and $\mathbb{N} T_{2}$.

This proposition means that the only way to show that an extension in $\mathbb{P}^{n+1}$ is an s.t.c.i. via gluing is to apply the technique to a projective monomial curve in $\mathbb{P}^{n}$. Thus we discuss the case where $T_{1}$ is a singleton. But if $T_{1}$ is $\left\{\left(m_{n+1}, 0\right)\right\}$ or $\left\{\left(0, m_{n+1}\right)\right\}$, then $\mathbb{N} T_{1} \cap \mathbb{N} T_{2}=\{(0,0)\}$. So it is sufficient to deal with the case where $T_{1}$ is of the form $\left\{\left(m_{n+1}-m_{i}, m_{i}\right)\right\}$, for some $i \in\{1, \ldots, n\}$.

From now on, $\Delta_{i}$ denotes the greatest common divisor of the positive integers $m_{1}, \ldots, \widehat{m_{i}}, \ldots, m_{n+1}\left(m_{i}\right.$ is omitted $)$, for $i=1, \ldots, n$. Note that we have $\operatorname{gcd}\left(\Delta_{i}, m_{i}\right)=1$, for all $i=1, \ldots, n$, since $\operatorname{gcd}\left(m_{1}, \ldots, m_{n+1}\right)=1$.

Proposition 3.2. If $T_{1}=\left\{\left(m_{n+1}-m_{i_{0}}, m_{i_{0}}\right)\right\}$ for some fixed $i_{0} \in\{1, \ldots, n\}$, then $\mathbb{N} T$ is a gluing of $\mathbb{N} T_{1}$ and $\mathbb{N} T_{2}$ if and only if there exist non-negative integers $d_{j}$, for $j=1, \ldots, \hat{i}_{0}, \ldots, n+1$, satisfying the following two conditions: 
(I) $\Delta_{i_{0}} m_{i_{0}}=\sum_{\substack{j=1 \\ j \neq i_{0}}}^{n+1} d_{j} m_{j}$ and

(II) $\Delta_{i_{0}} \geq \sum_{\substack{j=1 \\ j \neq i_{0}}}^{n+1} d_{j}$

Proof. Let $\alpha=\Delta_{i_{0}}\left(m_{n+1}-m_{i_{0}}, m_{i_{0}}\right)$. We first show that $\mathbb{Z} T_{1} \cap \mathbb{Z} T_{2}=\mathbb{Z} \alpha$. Since $\Delta_{i_{0}}=\operatorname{gcd}\left(m_{1}, \ldots, \widehat{m_{i_{0}}}, \ldots, m_{n+1}\right)$, there are $z_{j} \in \mathbb{Z}$, for $j=1, \ldots, \widehat{i_{0}}, \ldots, n+1$, such that $\Delta_{i_{0}}=\sum_{j \neq i_{0}} z_{j} m_{j}$. So $\Delta_{i_{0}} m_{i_{0}}=\sum_{j \neq i_{0}} m_{i_{0}} z_{j} m_{j}$, which implies that

$\Delta_{i_{0}}\left(m_{n+1}-m_{i_{0}}, m_{i_{0}}\right)=\sum_{j \neq i_{0}} m_{i_{0}} z_{j}\left(m_{n+1}-m_{j}, m_{j}\right)+\left(\Delta_{i_{0}}-\sum_{j \neq i_{0}} m_{i_{0}} z_{j}\right)\left(m_{n+1}, 0\right)$.

Thus $\alpha=\Delta_{i_{0}}\left(m_{n+1}-m_{i_{0}}, m_{i_{0}}\right) \in \mathbb{Z} T_{1} \cap \mathbb{Z} T_{2}$, implying $\mathbb{Z} \alpha \subseteq \mathbb{Z} T_{1} \cap \mathbb{Z} T_{2}$.

For the converse inclusion, take $c\left(m_{n+1}-m_{i_{0}}, m_{i_{0}}\right) \in \mathbb{Z} T_{1} \cap \mathbb{Z} T_{2}$, for some $c \in \mathbb{Z}$. Then, obviously we have $c\left(m_{n+1}-m_{i_{0}}, m_{i_{0}}\right) \in \mathbb{Z} T_{2}$, which implies that $c m_{i_{0}} \in \mathbb{Z}\left(\left\{m_{1}, \ldots, \widehat{m_{i_{0}}}, \ldots, m_{n+1}\right\}\right)=\mathbb{Z} \Delta_{i_{0}}$. So $\Delta_{i_{0}}$ divides $c m_{i_{0}}$. If $\Delta_{i_{0}}>1$, then $\Delta_{i_{0}}$ divides $c$, since it does not divide $m_{i_{0}}$ (remember that $\left.\operatorname{gcd}\left(\Delta_{i_{0}}, m_{i_{0}}\right)=1\right)$. If $\Delta_{i_{0}}=1$, obviously $\Delta_{i_{0}}$ divides $c$. Thus, $c\left(m_{n+1}-m_{i_{0}}, m_{i_{0}}\right)$ is a multiple of $\alpha$ and $\mathbb{Z} T_{1} \cap \mathbb{Z} T_{2} \subseteq \mathbb{Z} \alpha$

Since $\mathbb{Z} T_{1} \cap \mathbb{Z} T_{2}=\mathbb{Z} \alpha$, it will follow by definition that $\mathbb{N} T$ is a gluing of $\mathbb{N} T_{1}$ and $\mathbb{N} T_{2}$ if and only if $\alpha \in \mathbb{N} T_{1} \cap \mathbb{N} T_{2}$. But, if $\alpha \in \mathbb{N} T_{1} \cap \mathbb{N} T_{2}$, then there exist non-negative integers $d_{j}$ and $d$ for which we have

$$
\begin{aligned}
\Delta_{i_{0}}\left(m_{n+1}-m_{i_{0}}, m_{i_{0}}\right) & =\sum_{j \neq i_{0}} d_{j}\left(m_{n+1}-m_{j}, m_{j}\right)+d\left(m_{n+1}, 0\right), \\
\left(\Delta_{i_{0}} m_{n+1}-\Delta_{i_{0}} m_{i_{0}}, \Delta_{i_{0}} m_{i_{0}}\right) & =\left(\left[d+\sum_{j \neq i_{0}} d_{j}\right] m_{n+1}-\sum_{j \neq i_{0}} d_{j} m_{j}, \sum_{j \neq i_{0}} d_{j} m_{j}\right) .
\end{aligned}
$$

Thus, $\Delta_{i_{0}} m_{i_{0}}=\sum_{j \neq i_{0}} d_{j} m_{j}$ and $d=\Delta_{i_{0}}-\sum_{j \neq i_{0}} d_{j}$. Since $d \geq 0$, we see that the conditions (I) and (II) hold. On the other hand, if (I) and (II) hold, then we observe that $\alpha \in \mathbb{N} T_{1} \cap \mathbb{N} T_{2}$, by the equalities above. Thus, the condition $\alpha \in \mathbb{N} T_{1} \cap \mathbb{N} T_{2}$ is equivalent to the existence of the non-negative integers $d_{j}$ satisfying (I) and (II).

As a direct consequence of Proposition 3.2, we get the following:

Corollary 3.3. If $\Delta_{i_{0}}=1$, for some fixed $i_{0} \in\{1, \ldots, n\}$, then $\mathbb{N} T$ cannot be obtained as a gluing of $\mathbb{N} T_{1}$ and $\mathbb{N} T_{2}$, where $T_{1}=\left\{\left(m_{n+1}-m_{i_{0}}, m_{i_{0}}\right)\right\}$ and $T_{2}=$ $T-T_{1}$.

Proof. We apply Proposition 3.2 . If (I) does not hold, we are done. If (I) does hold, then we have two cases: either $\sum_{\substack{j=1 \\ j \neq i_{0}}}^{n+1} d_{j}=1$ or $\sum_{\substack{j=1 \\ j \neq i_{0}}}^{n+1} d_{j}>1$. The first case forces $m_{i_{0}}=m_{j}$ for some $j \neq i_{0}$, from (I), but this contradicts the way we choose $m_{i}$ 's. The second case causes (II) to fail, as $\Delta_{i_{0}}=1$.

Example 3.4. If we consider the curve $\bar{C}(2,3,4,8) \subset \mathbb{P}^{4}$ and take $i_{0}=2$, then the conditions (I) and (II) of the above proposition hold. Thus this curve can be obtained by gluing. 
However, if we consider the monomial curve $\bar{C}(2,4,7,8) \subset \mathbb{P}^{4}$, then for every choice of $i_{0}$, either $\Delta_{i_{0}}=1$ or else condition (II) of the above proposition fails. Hence this curve cannot be obtained by gluing.

Corollary 3.5. Let $\bar{C}_{\ell, m} \subset \mathbb{P}^{n+1}$ be a bad extension of $\bar{C}=\bar{C}\left(m_{1}, \ldots, m_{n}\right)$, i.e. $\ell \geq \delta(m)$. If $\bar{C}$ is an s.t.c.i. on the hypersurfaces $f_{1}=\cdots=f_{n-1}=0$, then $\bar{C}_{\ell, m}$ can be shown to be an s.t.c.i. on the hypersurfaces $f_{1}=\cdots=f_{n-1}=0$ and $F=x_{n+1}^{\ell}-x_{0}^{\ell-\delta(m)} x_{1}^{s_{1}} \cdots x_{n}^{s_{n}}=0$ by the technique of gluing, where $m=$ $s_{1} m_{1}+\cdots+s_{n} m_{n}$ and $s_{1}+\cdots+s_{n}=\delta(m)$.

Proof. Since $m_{1}<\cdots<m_{n}$ and $m=s_{1} m_{1}+\cdots+s_{n} m_{n} \leq \delta(m) m_{n} \leq \ell m_{n}$, it follows that $\ell m_{n}$ is the biggest number among $\left\{\ell m_{1}, \ldots, \ell m_{n}, m\right\}$. The extension $\bar{C}_{\ell, m}$ corresponds to the semigroup $\mathbb{N} T$, where $T=T_{1} \cup T_{2}, T_{1}=\left\{\left(\ell m_{n}-m, m\right)\right\}$ and $T_{2}=\left\{\left(\ell m_{n}, 0\right),\left(\ell m_{n}-\ell m_{1}, \ell m_{1}\right), \ldots,\left(\ell m_{n}-\ell m_{n-1}, \ell m_{n-1}\right),\left(0, \ell m_{n}\right)\right\}$. Since $\operatorname{gcd}\left(\ell m_{1}, \ldots, \ell m_{n}\right)=\ell, \ell m=s_{1}\left(\ell m_{1}\right)+\cdots+s_{n}\left(\ell m_{n}\right)$ and $\ell \geq \delta(m), \mathbb{N} T$ is a gluing of $\mathbb{N} T_{1}$ and $\mathbb{N} T_{2}$, by Proposition 3.2. Since $I(T)=I\left(T_{1}\right)+I\left(T_{2}\right)+(F)$, the claim follows from [16, Theorem 2].

\section{The main Results}

Since bad extensions are shown to be an s.t.c.i. by the technique of gluing (see Corollary 3.5 above), we study nice extensions of monomial curves in this section. By using the theory developed in the previous section, one can check which of these extensions can be obtained by the technique of gluing semigroups.

Throughout this section we will assume that

- $\bar{C}=\bar{C}\left(m_{1}, \ldots, m_{n}\right) \subset \mathbb{P}^{n}$ is an s.t.c.i. on $f_{1}=\cdots=f_{n-1}=0$.

- $m=s_{1} m_{1}+\cdots+s_{n} m_{n}$ for some nonnegative integers $s_{1}, \ldots, s_{n}$ such that $s_{1}+\cdots+s_{n}=\delta(m)$.

- $\ell$ is a positive integer with $\operatorname{gcd}(\ell, m)=1$.

- $\delta(m)>\ell$.

Remark 4.1. Since $\bar{C}$ is an s.t.c.i. on $f_{1}=\cdots=f_{n-1}=0$, its affine part $C$ is an s.t.c.i. on $g_{1}=\cdots=g_{n-1}=0$, where $g_{i}\left(x_{1}, \ldots, x_{n}\right)=f_{i}\left(1, x_{1}, \ldots, x_{n}\right)$ is the dehomogenization of $f_{i}, i=1, \ldots, n-1$. It follows from Theorem 2.3 that $C_{\ell, m}$ is an s.t.c.i. on the hypersurfaces $g_{i}=0$ and $G=x_{1}{ }^{s_{1}} \cdots x_{n}{ }^{s_{n}}-x_{n+1}^{\ell}=0$. So the ideal of the affine curve $C_{\ell, m}$ contains $g_{i}$ 's and $G$. Hence the ideal of the projective closure of $C_{\ell, m}$ must contain (at least) $f_{i}$ 's and $F$, where $F$ is the homogenization of $G$. Now, since $f_{1}, \ldots, f_{n-1}, F \in I\left(\bar{C}_{\ell, m}\right)$, we always have $\bar{C}_{\ell, m} \subseteq Z\left(f_{1}, \ldots, f_{n-1}, F\right)$.

4.1. The case where $f_{i}$ 's are general, but $m$ is special. In this section we assume that $m$ is a multiple of $m_{n}$; i.e. $m=s_{n} m_{n}$, where $s_{n}$ is a positive integer. Note that $\left(s_{1}, \ldots, s_{n-1}\right)=(0, \ldots, 0)$ and $\delta(m)=s_{n}$ in this case.

Theorem 4.2. Let $\bar{C} \subset \mathbb{P}^{n}$ be an s.t.c.i. on the hypersurfaces $f_{1}=\cdots=f_{n-1}=0$, $\operatorname{gcd}\left(\ell, s_{n} m_{n}\right)=1$ and $s_{n}>\ell$. Then the nice extensions $\bar{C}_{\ell, s_{n} m_{n}}$ in $\mathbb{P}^{n+1}$ are s.t.c.i.'s on $f_{1}=\cdots=f_{n-1}=F=0$, where $F=x_{n}^{s_{n}}-x_{0}^{s_{n}-\ell} x_{n+1}^{\ell}$.

Proof. The fact that these nice extensions are s.t.c.i.'s can be seen easily by [14, Theorem 3.4] taking $b_{1}=m_{1}, \ldots, b_{n-1}=m_{n-1}, d=m_{n}$ and $k=\left(s_{n}-\ell\right) m_{n}$. In addition to this, we provide here the equation of the binomial hypersurface $F=0$ on which these extensions lie as s.t.c.i. monomial curves. 
Since $\bar{C}_{\ell, s_{n} m_{n}} \subseteq Z\left(f_{1}, \ldots, f_{n-1}, F\right)$, we need to get the converse inclusion. Take a point $P=\left(p_{0}, \ldots, p_{n}, p_{n+1}\right) \in Z\left(f_{1}, \ldots, f_{n-1}, F\right)$. Then, since $f_{i} \in K\left[x_{0}, \ldots, x_{n}\right]$, we have $f_{i}(P)=f_{i}\left(p_{0}, \ldots, p_{n}\right)=0$, for all $i=1, \ldots, n-1$. Since $Z\left(f_{1}, \ldots, f_{n-1}\right)=$ $\bar{C}$ in $\mathbb{P}^{n}$ by assumption, the last observation implies that

$$
\left(p_{0}, \ldots, p_{n}\right)=\left(u^{m_{n}}, u^{m_{n}-m_{1}} v^{m_{1}}, \ldots, u^{m_{n}-m_{n-1}} v^{m_{n-1}}, v^{m_{n}}\right) .
$$

If $p_{0}=0$, then $u=0$, yielding that $\left(p_{0}, \ldots, p_{n-1}, p_{n}\right)=\left(0, \ldots, 0, p_{n}\right)$. Since $s_{n}>\ell$, we also have $p_{n}=0$ by $F\left(0, \ldots, 0, p_{n}, p_{n+1}\right)=p_{n}{ }^{s_{n}}-p_{0}^{s_{n}-\ell} p_{n+1}^{\ell}=0$. So we observe that $\left(p_{0}, \ldots, p_{n}, p_{n+1}\right)=(0, \ldots, 0,1)$, which is on the curve $\bar{C}_{\ell, s_{n} m_{n}}$. If $p_{0}=1$, then $\left(1, p_{1}, \ldots, p_{n}, p_{n+1}\right) \in Z\left(g_{1}, \ldots, g_{n-1}, G\right)$ by the assumption, where $g_{i}$ and $G$ are polynomials defined in Remark 4.1. Since $C_{\ell, s_{n} m_{n}}$ is an s.t.c.i. on the hypersurfaces $g_{1}=\cdots=g_{n-1}=0$ and $G=0$, it follows that $\left(1, p_{1}, \ldots, p_{n}, p_{n+1}\right) \in$ $C_{\ell, s_{n} m_{n}} \subset \bar{C}_{\ell, s_{n} m_{n}}$.

4.2. The case where $f_{i}$ 's are special and $m$ is general. Assume now that $m$ is not a multiple of $m_{n}$, i.e. $\left(s_{1}, \ldots, s_{n-1}\right) \neq(0, \ldots, 0)$. Recall that we choose $s_{1}, \ldots, s_{n}$ in the representation of $m=s_{1} m_{1}+\cdots+s_{n} m_{n}$ in such a way that $s_{1}+\cdots+s_{n}$ is minimum, i.e. $s_{1}+\cdots+s_{n}=\delta(m)$. First we prove a lemma where no restriction on the $f_{i}$ is required.

Lemma 4.3. Let $\bar{C} \subset \mathbb{P}^{n}$ be an s.t.c.i. on $f_{1}=\cdots=f_{n-1}=0$ and $\delta(m)>\ell$. Then $Z\left(f_{1}, \ldots, f_{n-1}, F\right)=\bar{C}_{\ell, m} \cup L \subset \mathbb{P}^{n+1}$, where $F=x_{1}^{s_{1}} \cdots x_{n}{ }^{s_{n}}-x_{0}^{\delta(m)-\ell} x_{n+1}^{\ell}$ and $L$ is the line $x_{0}=\cdots=x_{n-1}=0$.

Proof. We first prove $\bar{C}_{\ell, m} \cup L \subseteq Z\left(f_{1}, \ldots, f_{n-1}, F\right)$. By Remark4.1, it is sufficient to see that $L \subseteq Z\left(f_{1}, \ldots, f_{n-1}, F\right)$. For this, we take a point $P=\left(p_{0}, \ldots, p_{n+1}\right)$ on the line $L$, i.e., $P=\left(0, \ldots, 0, p_{n}, p_{n+1}\right)$. Since $\left(s_{1}, \ldots, s_{n-1}\right) \neq(0, \ldots, 0)$ and $\delta(m)>\ell$, we see that $F(P)=0$. Letting $v \in K$ be any $m_{n}$-th root of $p_{n}$, we get $\left(0, \ldots, 0, p_{n}\right)=\left(0, \ldots, 0, v^{m_{n}}\right) \in \bar{C}=Z\left(f_{1}, \ldots, f_{n-1}\right)$. Since the polynomials $f_{i}$ are in $K\left[x_{0}, \ldots, x_{n}\right]$, it follows that $f_{i}(P)=f_{i}\left(0, \ldots, 0, p_{n}\right)=0$, for all $i=1, \ldots, n-1$. Thus $P \in Z\left(f_{1}, \ldots, f_{n-1}, F\right)$.

For the converse inclusion, take $P=\left(p_{0}, \ldots, p_{n}, p_{n+1}\right) \in Z\left(f_{1}, \ldots, f_{n-1}, F\right)$. Then, for all $i=0, \ldots, n-1$, we get $f_{i}\left(p_{0}, \ldots, p_{n}\right)=f_{i}(P)=0$, implying that

$$
\left(p_{0}, \ldots, p_{n}\right)=\left(u^{m_{n}}, u^{m_{n}-m_{1}} v^{m_{1}}, \ldots, u^{m_{n}-m_{n-1}} v^{m_{n-1}}, v^{m_{n}}\right) .
$$

If $p_{0}=0$, then $u=0$, yielding that $\left(p_{0}, \ldots, p_{n}\right)=\left(0, \ldots, 0, p_{n}\right)$. Thus, we get $P=\left(p_{0}, \ldots, p_{n}, p_{n+1}\right)=\left(0, \ldots, 0, p_{n}, p_{n+1}\right) \in L$. If $p_{0}=1$, then by assumption we know that $P=\left(1, p_{1}, \ldots, p_{n}, p_{n+1}\right) \in Z\left(g_{1}, \ldots, g_{n-1}, G\right)$. Since $C_{\ell, m}$ is an s.t.c.i. on the hypersurfaces $g_{1}=\cdots=g_{n-1}=0$ and $G=0$, it follows that $P=\left(1, p_{1}, \ldots, p_{n}, p_{n+1}\right) \in C_{\ell, m} \subset \bar{C}_{\ell, m}$.

To get rid of $L$ in the intersection of the hypersurfaces $f_{1}=\cdots=f_{n-1}=0$ and $F=0$, we modify the $F=x_{1}{ }^{s_{1}} \cdots x_{n}{ }^{s_{n}}-x_{0}^{\delta(m)-\ell} x_{n+1}^{\ell}$ of Lemma 4.3, as in the work of Bresinsky (see [4]), for some special choice of $f_{1}, \ldots, f_{n-1}$. In this way we construct a new polynomial $F^{*}$ from $F$ such that $Z\left(f_{1}, \ldots, f_{n-1}, F^{*}\right)=\bar{C}_{\ell, m}$, where $F^{*}$ is a polynomial of the form

$$
F^{*}=x_{n}^{\alpha}+x_{0}^{\beta} H\left(x_{0}, \ldots, x_{n+1}\right),
$$

where $\beta$ is a positive integer.

Note that when $x_{0}=0$, the vanishing of $F^{*}$ implies that $x_{n}=0$. It follows from the last part of the proof of Lemma 4.3 that this property of $F^{*}$ ensures that we 
have a point at infinity in the intersection of $f_{1}=\cdots=f_{n-1}=0$ and $F^{*}=0$ instead of a line.

The construction of $F^{*}$ can be described as follows. We first assume that $f_{i}=$ $x_{i}^{a_{i}}-x_{0}^{a_{i}-b_{i}} x_{n}^{b_{i}}=0$, where $a_{i}>b_{i}$ are positive integers, for all $i=1, \ldots, n-1$. Let $p=a_{1} \cdots a_{n-1}$ and $p_{i}=\frac{b_{i}}{a_{i}} p$, for $i=1, \ldots, n-1$. Take the $p$-th power of $F$, and for every occurrence of $x_{i}^{a_{i}}$ substitute $x_{0}^{a_{i}-b_{i}} x_{n}^{b_{i}}$ for all $i=1, \ldots, n-1$. Then we have

$$
\begin{aligned}
F^{p} & =x_{0}^{\gamma} x_{n}^{\alpha}+x_{0}^{\delta(m)-\ell} H\left(x_{0}, \ldots, x_{n+1}\right) \bmod \left(f_{1}, \ldots, f_{n-1}\right) \\
& =x_{0}^{\gamma}\left[x_{n}^{\alpha}+x_{0}^{\delta(m)-\ell-\gamma} H\left(x_{0}, \ldots, x_{n+1}\right)\right] \bmod \left(f_{1}, \ldots, f_{n-1}\right),
\end{aligned}
$$

where $\gamma=\sum_{i=1}^{n-1}\left(p-p_{i}\right) s_{i}, \alpha=p s_{n}+\sum_{i=1}^{n-1} p_{i} s_{i}$ and $H$ is a polynomial. Letting

$$
F^{*}\left(x_{0}, \ldots, x_{n+1}\right)=x_{n}^{\alpha}+x_{0}^{\delta(m)-\ell-\gamma} H\left(x_{0}, \ldots, x_{n+1}\right),
$$

we observe that

$$
F^{p}\left(x_{0}, \ldots, x_{n+1}\right)=x_{0}^{\gamma} F^{*}\left(x_{0}, \ldots, x_{n+1}\right) \bmod \left(f_{1}, \ldots, f_{n-1}\right) .
$$

Recall that $m$ is an element of the numerical semigroup generated by $m_{1}, \ldots, m_{n}$; i.e. $m=s_{1} m_{1}+\cdots+s_{n} m_{n}$ with $s_{1}+\cdots+s_{n}=\delta(m)$. If $m$ is large enough that $s_{n}>\ell+\sum_{i=1}^{n-1}\left(p-p_{i}-1\right) s_{i}$ (or equivalently $\delta(m)-\ell-\gamma>0$ ), then $F^{*}$ is the required polynomial. (Otherwise, $F^{*}$ may not be a polynomial.) Hence we conclude the following:

Theorem 4.4. Let $p, p_{i}, f_{i}$ and $F^{*}$ be as above. Assume that $m$ is chosen so that $s_{n}>\ell+\sum_{i=1}^{n-1}\left(p-p_{i}-1\right) s_{i}$. Then, for all $\ell<\delta(m)$ with $\operatorname{gcd}(\ell, m)=1$, the nice extensions $\bar{C}_{\ell, m} \subset \mathbb{P}^{n+1}$ are s.t.c.i.'s on $f_{1}=\cdots=f_{n-1}=0$ and $F^{*}=0$.

Proof. We will show that $\bar{C}_{\ell, m}$ is an s.t.c.i. on $f_{1}=\cdots=f_{n-1}=0$ and $F^{*}=0$. To do this, take a point $P=\left(p_{0}, \ldots, p_{n+1}\right) \in \bar{C}_{\ell, m}$. Then, $F(P)=0$ and $f_{i}(P)=0$, for all $i=1, \ldots, n-1$, since $Z\left(f_{1}, \ldots, f_{n-1}, F\right)=\bar{C}_{\ell, m} \cup L$, by Lemma 4.3. From equation (4.1) it follows that $F^{*}(P)=0$ or $p_{0}=0$. Since $P$ is a point on the monomial curve $\bar{C}_{\ell, m}$, it can be parameterized as follows:

$$
\left(u^{m}, u^{m-\ell m_{1}} v^{\ell m_{1}}, \ldots, u^{m-\ell m_{n}} v^{\ell m_{n}}, v^{m}\right) .
$$

So if $p_{0}=0$, we get $u=0$ and thus $p_{i}=0$, for all $i=1, \ldots, n$. Therefore $P=(0, \ldots, 0,1)$, and hence $F^{*}(P)=0$ in any case.

Conversely, let $P=\left(p_{0}, \ldots, p_{n+1}\right) \in Z\left(f_{1}, \ldots, f_{n-1}, F^{*}\right)$. If $p_{0}=0$, then $p_{i}=0$ by $f_{i}(P)=0$, for all $i=1, \ldots, n-1$. Since $\delta(m)-\ell-\gamma>0$, we have $p_{n}=0$ by $F^{*}(P)=0$. Thus $P=(0, \ldots, 0,1)$, which is always on the curve $\bar{C}_{\ell, m}$. If $p_{0}=1$, then $C$ is an s.t.c.i. on the hypersurfaces given by $g_{i}=x_{i}^{a_{i}}-x_{i+1}^{b_{i}}=0$, for $i=1, \ldots, n-1$, by the assumption. Hence, Theorem 2.3 implies that $C_{\ell, m}$ is an s.t.c.i. on $g_{1}=\cdots=g_{n-1}=0$ and $G=x_{1}{ }^{s_{1}} \cdots x_{n}{ }^{s_{n}}-x_{n+1}^{\ell}=0$. Thus $P=\left(1, p_{1}, \ldots, p_{n+1}\right) \in C_{\ell, m} \subset \bar{C}_{\ell, m}$.

Remark 4.5. The nice extensions in Theorem 4.4 can also be shown to be s.t.c.i.'s by using [14, Theorem 3.4]. But to show that the hypotheses of [14, Theorem 3.4] are satisfied by these extensions is much more difficult than the proof here. As a byproduct we also constructed here the hypersurface $F^{*}=0$ on which these nice extensions are s.t.c.i.'s. 
Example 4.6. We start with $\bar{C}=\bar{C}(3,4,6) \subset \mathbb{P}^{3}$. Let $\ell=1$ and $m=6 s+7$, for some positive integer $s$. Then $\delta(m)=s+2, s_{1}=s_{2}=1$ and $s_{3}=s$. Thus we get the nice extensions $\bar{C}_{1,6 s+7}=\bar{C}(3,4,6,6 s+7) \subset \mathbb{P}^{4}$. Since $\Delta_{1}=\operatorname{gcd}(4,6,6 s+7)=$ $1, \Delta_{2}=\operatorname{gcd}(3,6,6 s+7)=1$ and $\Delta_{3}=\operatorname{gcd}(3,4,6 s+7)=1$, it follows from Corollary 3.3 that these curves cannot be obtained by gluing. Using the software Macaulay, it is easy to see that the ideal of $\bar{C}_{1,6 s+7}$ is minimally generated by the polynomials

$$
\begin{aligned}
f_{1} & =x_{1}^{2}-x_{0} x_{3}, \\
f_{2} & =x_{2}^{3}-x_{0} x_{3}^{2}, \\
f_{3} & =x_{3}^{s+3}-x_{0}^{s-1} x_{1} x_{2}^{2} x_{4}, \\
f_{4} & =x_{2} x_{3}^{s+1}-x_{0}^{s} x_{1} x_{4}, \\
f_{5} & =x_{1} x_{3}^{s+2}-x_{0}^{s} x_{2}^{2} x_{4}, \\
F & =x_{1} x_{2} x_{3}^{s}-x_{0}^{s+1} x_{4} .
\end{aligned}
$$

Since $\bar{C}(3,4,6) \subset \mathbb{P}^{3}$ is an s.t.c.i. on the surfaces $f_{1}=0$ and $f_{2}=0$, it follows from Theorem 4.4 that $\bar{C}_{1,6 s+7}$ is an s.t.c.i. on $f_{1}=0, f_{2}=0$ and

$$
\begin{gathered}
F^{*}=x_{3}^{6 s+7}-6 x_{0}^{s-1} x_{1} x_{2}^{2} x_{3}^{5 s+4} x_{4}+15 x_{0}^{2 s} x_{2} x_{3}^{4 s+4} x_{4}^{2}-20 x_{0}^{3 s} x_{1} x_{3}^{3 s+3} x_{4}^{3} \\
+15 x_{0}^{4 s} x_{2}^{2} x_{3}^{2 s+1} x_{4}^{4}-6 x_{0}^{5 s} x_{1} x_{2} x_{3}^{s} x_{4}^{5}+x_{0}^{6 s+1} x_{4}^{6}=0,
\end{gathered}
$$

provided that $s>2$.

\section{VARIATIONS AND CONSEQUENCES OF THE MAIN RESUltS}

In this section, we give some consequences of Theorem 4.2, and hence all the notation is as in that theorem. We also include some theorems about nice extensions of projective monomial curves that are variations of Theorem 4.4

5.1. Consequences of Theorem 4.2, Since arithmetically Cohen-Macaulay monomial curves are s.t.c.i.'s in $\mathbb{P}^{3}$ (see [12]), we get the following corollary as a consequence of Theorem 4.2 .

Corollary 5.1. Let $\bar{C}\left(m_{1}, m_{2}, m_{3}\right)$ be an arithmetically Cohen-Macaulay monomial curve in $\mathbb{P}^{3}$. Let $m=s_{3} m_{3}, \operatorname{gcd}(\ell, m)=1$ and $\delta(m)=s_{3}>\ell$. Then the nice extensions $\bar{C}_{\ell, s_{3} m_{3}}=\bar{C}\left(\ell m_{1}, \ell m_{2}, \ell m_{3}, s_{3} m_{3}\right)$ are all s.t.c.i.'s in $\mathbb{P}^{4}$.

Remark 5.2. There are very few examples of s.t.c.i. monomial curves in $\mathbb{P}^{n}$, where $n>3$. We know that the rational normal curve $\bar{C}(1,2, \ldots, n)$ is an s.t.c.i. in $\mathbb{P}^{n}$, for any $n>0$ (see [11, 14]). Applying Theorem 4.2 to $\bar{C}(1,2, \ldots, n) \subset \mathbb{P}^{n}$, we can produce infinitely many new examples of s.t.c.i. monomial curves in $\mathbb{P}^{n+1}$ :

Corollary 5.3. For all positive integers $\ell, n$ and $s$ with $\operatorname{gcd}(\ell, s n)=1$, the monomial curves $\bar{C}(\ell, 2 \ell, \ldots, n \ell, s n) \subset \mathbb{P}^{n+1}$ are s.t.c.i.'s.

Proof. Let $m=s n$. Clearly $\delta(m)=s$. If $s \leq \ell$, then the curves $\bar{C}_{\ell, m}=$ $\bar{C}(\ell, 2 \ell, \ldots, n \ell, s n) \subset \mathbb{P}^{n+1}$ are bad extensions of $\bar{C}(1,2, \ldots, n) \subset \mathbb{P}^{n}$. Hence they are s.t.c.i.'s by Corollary 3.5. If $s>\ell$, then these curves are nice extensions of $\bar{C}(1,2, \ldots, n) \subset \mathbb{P}^{n}$. Therefore they are s.t.c.i.'s by Theorem 4.2 ,

In [10, all complete intersection (i.t.c.i.) lattice ideals are characterized by gluing semigroups. But, for a given projective monomial curve it is not easy to find two 
subsemigroups whose ideals are complete intersections. So, as another application of Theorem 4.2 we can produce infinitely many i.t.c.i. monomial curves:

Proposition 5.4. If $\bar{C} \subset \mathbb{P}^{n}$ is an i.t.c.i., then the nice extensions $\bar{C}_{\ell, s_{n} m_{n}} \subset \mathbb{P}^{n+1}$ are i.t.c.i.'s for all positive integers $\ell$ and $s_{n}$ with $s_{n}>\ell, \operatorname{gcd}\left(\ell, s_{n} m_{n}\right)=1$.

Proof. Since $\bar{C}$ is an s.t.c.i. on the binomial hypersurfaces $f_{1}=\cdots=f_{n-1}=0$, it follows from Theorem 4.2 that $\bar{C}_{\ell, s_{n} m_{n}}$ is an s.t.c.i. on $f_{1}=\cdots=f_{n-1}=0$ and $F\left(x_{0}, \ldots, x_{n+1}\right)=x_{n}^{s_{n}}-x_{0}^{s_{n}-\ell} x_{n+1}^{\ell}=0$. Since these are all binomial, the monomial curves $\bar{C}_{\ell, s_{n} m_{n}}$ are i.t.c.i.'s on the same hypersurfaces, by [2, Theorem 4].

Corollary 5.5. The monomial curves $\bar{C}\left(\ell m_{1}, \ell m_{2}, s_{2} m_{2}\right)$ are i.t.c.i.'s in $\mathbb{P}^{3}$, for all positive integers $m_{1}, m_{2}, \ell$ and $s_{2}$ with $s_{2}>\ell, \operatorname{gcd}\left(\ell, s_{2} m_{2}\right)=1$.

Proof. Let $m=s_{2} m_{2}$. Then $\delta(m)=s_{2}$ and $\bar{C}_{\ell, m}=\bar{C}\left(\ell m_{1}, \ell m_{2}, s_{2} m_{2}\right)$ is a nice extension of $\bar{C}\left(m_{1}, m_{2}\right)$, by the assumption $s_{2}>\ell$. Since $\bar{C}\left(m_{1}, m_{2}\right)$ is an i.t.c.i. on $x_{1}^{m_{2}}-x_{0}^{m_{2}-m_{1}} x_{2}^{m_{1}}=0$, it follows from Proposition 5.4 that the nice extensions $\bar{C}\left(\ell m_{1}, \ell m_{2}, s_{2} m_{2}\right)$ are i.t.c.i.'s on $x_{1}^{m_{2}}-x_{0}^{m_{2}-m_{1}} x_{2}^{m_{1}}=0$ and $x_{2}^{s_{2}}-x_{0}^{s_{2}-\ell} x_{3}^{\ell}=0$.

To produce infinitely many examples of i.t.c.i. curves, our method starts from just one i.t.c.i. curve, whereas the semigroup gluing method produces only one example starting from one i.t.c.i. The following example illustrates this point.

Example 5.6. From Corollary 5.5 , we know that $\bar{C}(1,2,4)$ is an i.t.c.i. on

$$
f_{1}=x_{1}^{2}-x_{0} x_{2}=0 \text { and } f_{2}=x_{2}^{2}-x_{0} x_{3}=0 .
$$

Take two positive integers $\ell$ and $s$ with $s>\ell, \operatorname{gcd}(\ell, 4 s)=1$. Then the monomial curves $\bar{C}(\ell, 2 \ell, 4 \ell, 4 s) \subset \mathbb{P}^{4}$ are nice extensions of $\bar{C}(1,2,4) \subset \mathbb{P}^{3}$. Thus, by Proposition 5.4, the monomial curves $\bar{C}(\ell, 2 \ell, 4 \ell, 4 s)$ are i.t.c.i.'s on

$$
f_{1}=x_{1}^{2}-x_{0} x_{2}=0, f_{2}=x_{2}^{2}-x_{0} x_{3}=0 \text { and } F=x_{3}^{s}-x_{0}^{s-\ell} x_{4}^{\ell}=0 .
$$

The nice extensions $\bar{C}(\ell, 2 \ell, 4 \ell, 4 s)$ can also be obtained by gluing subsemigroups generated by $T_{1}=\{(4 s-\ell, \ell)\}$ and $T_{2}=\{(4 s, 0),(4 s-2 \ell, 2 \ell),(4 s-4 \ell, 4 \ell),(0,4 s)\}$. But in this case one has to know that $\bar{C}(\ell, 2 \ell, 2 s)$ is an i.t.c.i. for each $\ell$ and $s$. In other words, starting with the fact that $\bar{C}(1,2,4)$ is an i.t.c.i., the gluing method can only produce $\bar{C}(1,2,4,8)$ as an i.t.c.i. monomial curve.

5.2. Variations of Theorem 4.4. Recall that our method starts with a monomial curve $\bar{C}=Z\left(f_{1}, \ldots, f_{n-1}\right)$ in $\mathbb{P}^{n}$ and produces infinitely many nice extensions $\bar{C}_{\ell, m}=Z\left(f_{1}, \ldots, f_{n-1}, F^{*}\right)$ in $\mathbb{P}^{n+1}$. Since the construction of $F^{*}$ depends on the choice of $f_{1}, \ldots, f_{n-1}$, it is possible to start with another curve $\bar{C}=Z\left(f_{1}, \ldots, f_{n-1}\right)$ in $\mathbb{P}^{n}$ and obtain new families of nice extensions. In this section we provide two examples of this sort. For instance, if we assume that $\bar{C}$ is an s.t.c.i. on the hypersurfaces $f_{i}=x_{i}^{a_{i}}-x_{0}^{a_{i}-b_{i}} x_{i+1}^{b_{i}}=0$, where $a_{i}>b_{i}$ are positive integers, $i=$ $1, \ldots, n-1$, then under some suitable conditions we obtain other families of s.t.c.i. nice extensions. Let $p=a_{1} \cdots a_{n-1}, q_{0}=b_{1} \cdots b_{n-1}$ and $q_{i}=a_{1} \cdots a_{i} b_{i+1} \cdots b_{n-1}$, $i=1, \ldots, n-2$. The first variation is the following: 
Theorem 5.7. Let $p, q_{0}, \ldots, q_{n-2}$ be as above. For all $m$ which give rise to $s_{n}>$ $\ell+\sum_{i=0}^{n-2}\left(p-q_{i}-1\right) s_{i+1}$ and for all $\ell$ with $\ell<\delta(m)$ and $\operatorname{gcd}(\ell, m)=1$, the nice extensions $\bar{C}_{\ell, m} \subset \mathbb{P}^{n+1}$ are s.t.c.i.'s on $f_{1}=\cdots=f_{n-1}=F^{*}=0$.

Now, we give another variation where $m=s_{i} m_{i}+s_{j} m_{j}$, for $i, j \in\{1, \ldots, n\}$. For notational convenience we take $i=1$ and $j=n$.

Theorem 5.8. Let $\bar{C} \subset \mathbb{P}^{n}$ be an s.t.c.i. on the hypersurfaces given by

$$
\begin{aligned}
f_{1} & =x_{1}^{a}-x_{0}^{a-b} x_{n}^{b}=0, \\
f_{i} & =x_{i}^{a_{i}}+x_{0}^{b_{i}} A\left(x_{1}, \ldots, x_{n}\right)+x_{1}^{c_{i}} B\left(x_{2}, \ldots, x_{n}\right)=0,
\end{aligned}
$$

where $a, b, a-b, a_{i}, b_{i}$, and $c_{i}$ are positive integers, for $i=2, \ldots, n-1$, and where $A$ and $B$ are some polynomials. For all $m$ which give rise to $s_{n}>\ell+(a-b-1) s_{1}$ and for all $\ell$ with $\ell<\delta(m)$ and $\operatorname{gcd}(\ell, m)=1$, the nice extensions $\bar{C}_{\ell, m} \subset \mathbb{P}^{n+1}$ are s.t.c.i.'s on $f_{1}=\cdots=f_{n-1}=F^{*}=0$.

\section{ACKNOWLEDGEMENTS}

The author would like to extend his sincere thanks to F. Arslan, Ö. Kişisel, M. Morales, S. Sertöz and A. Thoma for their numerous comments, and to the anonymous referee for suggestions which improved the final presentation of the paper.

\section{REFERENCES}

[1] F. Arslan, P. Mete, Hilbert functions of Gorenstein monomial curves, Proc. Amer. Math. Soc. 135 (2007) 1993-2002. MR2299471 (2007m:13023)

[2] M. Barile, M. Morales, A. Thoma, Set-theoretic complete intersections on binomials, Proc. Amer. Math. Soc. 130 (2002) 1893-1903. MR1896020 (2003f:14058)

[3] D. Bayer and M. Stillman, Macaulay, a system for computations in algebraic geometry and commutative algebra, 1992, available at www.math.columbia.edu/ bayer/Macaulay

[4] H. Bresinsky, Monomial space curves in $\mathbb{A}^{3}$ as set-theoretic complete intersections, Proc. Amer. Math. Soc. 75 (1979) 23-24. MR529205 (81g:14022)

[5] D. Eisenbud, E.G. Evans, Every algebraic set in $n$-space is the intersection of $n$ hypersurfaces, Inventiones Math. 19 (1973) 107-112. MR0327783 (48:6125)

[6] K. Eto, Set-theoretic complete intersection lattice ideals in monoid rings, Journal of Algebra 299 (2006) 689-706. MR2228334 (2007a:13012)

[7] A. Katsabekis, Projection of cones and the arithmetical rank of toric varieties, Journal of Pure and Applied Algebra 199 (2005) 133-147. MR2134297 (2005m:14094)

[8] T.T. Moh, Set-theoretic complete intersections, Proc. Amer. Math. Soc. 94 (1985) 217-220. MR784166 (86e:14026)

[9] M. Morales, Noetherian symbolic blow-ups, Journal of Algebra 140 (1991) 12-25. MR.1114901 (92c:13020)

[10] M. Morales and A. Thoma, Complete intersection lattice ideals, Journal of Algebra 284 (2005) 755-770. MR.2114578 (2005j:20076)

[11] L. Robbiano, G. Valla, On set-theoretic complete intersections in the projective space, Rend. Sem. Mat. Fis. Milano LIII (1983) 333-346. MR858508 (87k:14057)

[12] L. Robbiano, G. Valla, Some curves in $\mathbb{P}^{3}$ are set-theoretic complete intersections, Algebraic geometry-open problems, Proceedings Ravello 1982, Lecture Notes in Mathematics, Vol. 997 (Springer, New York, 1983) 391-399. MR714759 (84m:14059)

[13] J.C. Rosales, On presentations of subsemigroups of $\mathbb{N}^{n}$, Semigroup Forum 55 (1997) 152-159. MR.1457760 (98h:20104)

[14] A. Thoma, On the set-theoretic complete intersection problem for monomial curves in $\mathbb{A}^{n}$ and $\mathbb{P}^{n}$, Journal of Pure and Applied Algebra 104 (1995) 333-344. MR1361579 (96h:13031) 
[15] A. Thoma, Affine semigroup rings and monomial varieties, Communications in Algebra 24(7) (1996) 2463-2471. MR.1390384 (97c:20096)

[16] A. Thoma, Construction of set-theoretic complete intersections via semigroup gluing, Contributions to Algebra and Geometry 41(1) (2000) 195-198. MR.1745589 (2001h:14059)

Department of Mathematics, Atilim University, 06836 Ankara, Turkey

E-mail address: mesut@atilim.edu.tr 